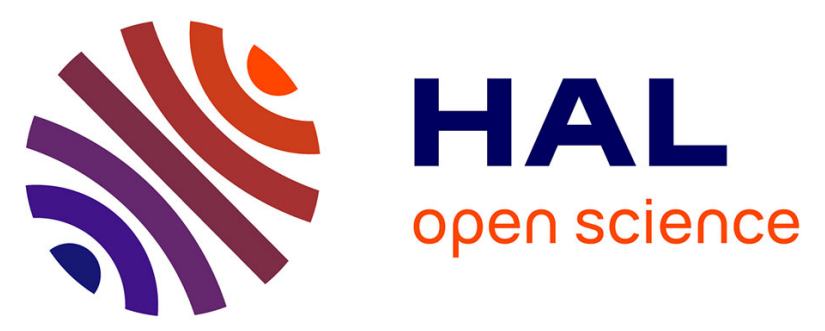

\title{
Reduced Order Modelling for efficient numerical optimisation of a hot-wall Chemical Vapour Deposition reactor
}

Domenico Borzacchiello, Jose Aguado, Francisco Chinesta

\section{- To cite this version:}

Domenico Borzacchiello, Jose Aguado, Francisco Chinesta. Reduced Order Modelling for efficient numerical optimisation of a hot-wall Chemical Vapour Deposition reactor. International Journal of Numerical Methods for Heat and Fluid Flow, 2016. hal-01925857

\section{HAL Id: hal-01925857 \\ https://hal.science/hal-01925857}

Submitted on 18 Nov 2018

HAL is a multi-disciplinary open access archive for the deposit and dissemination of scientific research documents, whether they are published or not. The documents may come from teaching and research institutions in France or abroad, or from public or private research centers.
L'archive ouverte pluridisciplinaire HAL, est destinée au dépôt et à la diffusion de documents scientifiques de niveau recherche, publiés ou non, émanant des établissements d'enseignement et de recherche français ou étrangers, des laboratoires publics ou privés. 


\title{
Reduced Order Modelling for efficient numerical optimisation of a hot-wall Chemical Vapour Deposition reactor
}

\author{
Domenico Borzacchiello José V. Aguado \\ Francisco Chinesta
}

\begin{abstract}
This paper presents a reduced order computational strategy for multiphysics simulation involving fluid flow, electrodynamics and heat transfer in a hot-wall Chemical Vapour Deposition (CVD) reactor. The main goal is to produce a multi-parametric solution for fast exploration of the design space in order to perform numerical prototyping and process optimisation. Different reduced order techniques are applied. In particular the Proper Generalized Decomposition (PGD) is used to solve the parametrised heat transfer equation in a five-dimensional space. The solution is provided in a compact separated-variable format allowing a fast evaluation of the process-specific quantities of interest that are involved in the optimisation algorithm.
\end{abstract}

Reduced Order Modelling; Multi-physics; Process Optimisation; Numerical Prototyping; Chemical Vapour Deposition; Proper Generalized Decomposition; High Dimensional Parametric PDE

\section{Introduction}

\section{$1.1 \quad$ Background}

Numerical optimisation and prototyping emerge as attractive tools to manage the design process in a variety of material forming applications. Before committing to produce a physical prototype, virtual models are useful to discern unfeasible solutions from the ones of practical interest using the tools of numerical simulation and automated design [1. In most cases, it is impossible to set up a global and exhaustive optimisation process at an early design stage, because some of the process variables may still not be defined. The main concern is therefore to evaluate different alternatives as quickly as possible and define a first concept that serves as a starting point for further optimisation. The pertinency of the physical model can be also assessed based on the virtual prototype and refined if needed. 


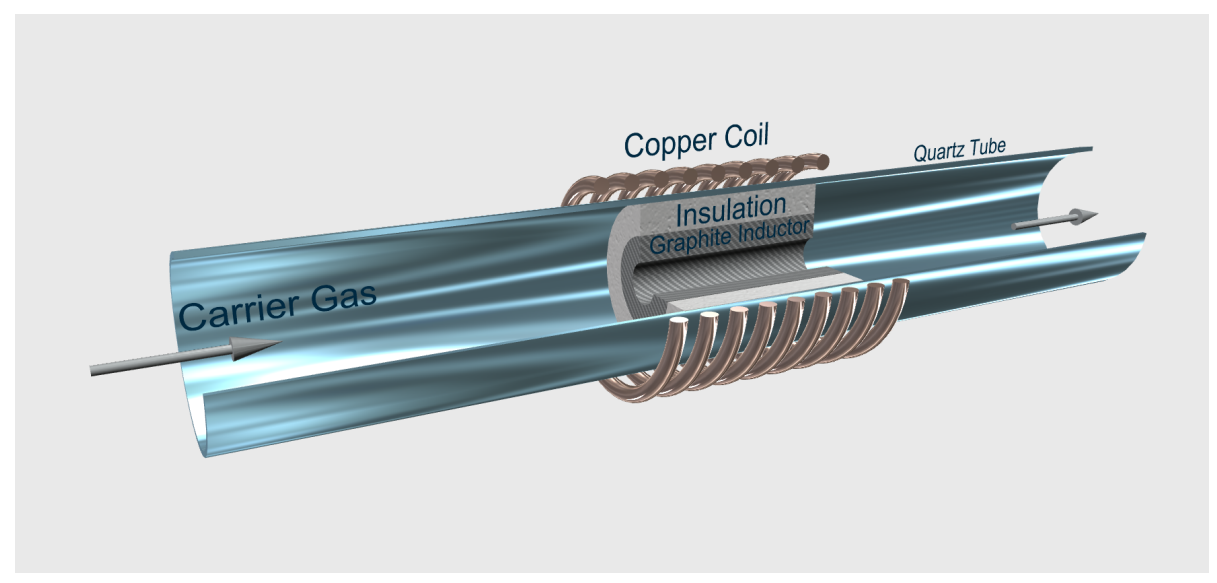

Figure 1: Two-dimensional axisymmetric schematics of the hot wall CVD reactor.

Optimisation is usually performed heuristically by human operators and is mostly based on the level of acquired experience. In material forming industry, optimisation of a new process often involves multi-physics and complex models. How ever much simplified the assumed models are, these are often unsolvable by sole intuition and experience. Numerical simulation-based design can offer a valid alternative in this case.

In this paper we turn our attention to the design of a horizontal hot-wall Chemical Vapour Deposition (CVD) reactor. This is used in many technological areas, including microelectronics, protective and decorative coatings, semiconductor films and carbon nano-fibres [2, 3. CVD technology involves the formation of a thin solid film on a substrate material by a chemical reaction of vapour-phase precursors. The deposition of the solid phase is controlled through the temperature field on the substrate where the film is grown. A simple schematic drawing of a reactor is shown in Fig. 11. The substrate is placed onto a hollow cylindrical graphite susceptor, which is inductively heated by an AC flowing through a copper coil. The susceptor is coated by insulation, and the whole chamber is enclosed in a quartz cylinder. The precursor gases that react with the substrate are diluted in a carrier gas which, in turn, also removes the by-products of the reaction.

\subsection{Numerical simulation of Chemical Vapour Deposition}

Numerical simulation of the CVD generally follows two approaches: the first is based on Molecular Dynamics and Monte Carlo Simulation [4, 5, 6, 17, 8, 9, 10, and is aimed to a microscopic description of chemical process responsible of the surface coating and the film growth. The second possible approach is based on Computational Fluid Dynamics (CFD) and describes the process in terms of macroscopic quantities like the fluid density, specific momentum and spe- 
cific energy, as well as species concentrations in the fluid. Among the vast literature available most of the models assume incompressible, laminar and axisymmetric flow [11, 12, 13]. The flow simulation includes heat transfer and advection-diffusion-reaction equations for the individual species. In 14 plasma physics and electrodynamics are also simulated and compressibility effects taken into account in the simulations of Plasma Enhanced CVD. In [15] the chemical composition is assumed to have little influence on the thermal and mechanical properties of the fluid, therefore the model only includes incompressible laminar flow, heat transfer and electrodynamics since inductive heating is taken into account.

Simulations are performed 2D axisymmetric flow and in 3D in order to account for buoyancy effects. In most of the works the objective of the design optimisation is related to the temperature field uniformity on the substrate where the film is grown [16, 15, 17] or, as in the case of multichannel structures, to the flow repartition and thickness uniformity of the epitaxial film [18.

All the above mentioned models rely on nonlinear coupling between the different physics in the sense that material parameters (mechanical and thermal properties) are considered as nonlinear functions of the temperature. The equations are discretised using Finite Volume or Finite Element techniques and solutions are found through nonlinear iterative solvers. A lot of emphasis is put on the necessity of integrating different existing codes into a single multiphysics solver [11, 12, 15, 13. This approach is generally time-consuming, and therefore unfit for the purpose of quickly validating or discarding a proposed solution, especially when screening numerous parameter combinations.

\subsection{Reduced Order Modelling}

Numerical optimisation requires fast reliable estimators rather than refined simulations. This implies that some approximations be accepted at this stage both in the formulation of the mathematical models and the accuracy of the numerical solutions. The former involves simplifying assumptions concerning the geometry and the degree of coupling between the different physics involved, while the latter requires numerical solvers that are capable to compute compact and inexpensive approximations of multidimensional solutions. In this context, Reduced Order Modelling (ROM) methods find an interesting and innovative field of application.

ROM exploits the correlations that naturally exist between the degrees of freedom of a numerical model and reduces the computational complexity of the problem by giving a lower dimensional representation of the solution. Different strategies are possible. A posteriori methods like Proper Orthogonal Decomposition (POD) [19, 20] and Reduced Basis Method (RBM) [21, 22, need of training simulations to discover the true dimensionality of the problem. The reduced model is then generally built using standard Galerkin projection techniques. On the contrary, a priori methods, like the Proper Generalized Decomposition (PGD) [23, 24, 25, 26, do not require any training simulation as they discover the true dimensionality of the problem within the computation of the solution. 
Previous attempts to ROM simulation based design are found in [27] where the a thermo-kinetics reduced model is built using POD, and in 28, 29], where reactive, incompressible laminar flow models are reduced using POD for automated control of the CVD process.

\subsection{Contributions}

In this work a priori ROM is used. A multi-parametric solution is explicitly obtained as a function of the gas flow rate, electrical input power and AC frequency. These parameters are no longer regarded as inputs of the simulation but are treated as extra-coordinates defining a higher dimensional computational domain. The use of separated representations ensures low computational complexity and storage requirements.

We show how parametrised equations can be solved in an offline stage using PGD (for the electrodynamics and heat transfer problems) and a hierarchical basis collocation method (for the flow problem). As the multi-parametric description of the numerical prototype behaviour is available, we proceed to illustrate how process optimisation can be efficiently performed in the online stage without further computational effort.

The rest of this paper is structured as follows: in section 2 we present the equations governing the physics of the problem. In section 3 the numerical methods are briefly reviewed and results are presented, while further details on the formulation and implementation are provided in Appendices $\mathrm{A}$ and $\mathrm{B}$. In section 4 we show how, with the proposed approach, optimisation can be considered as simple post-processing of the parametric solution. Finally conclusions are drawn in section 5 .

\section{Governing equations}

In this section we revisit a standard multi-physics modelling of the CVD reactor from [15] which is used to build the reduced model in next sections. Since our objective is to develop a simulation tool for evaluating the performance of numerical prototypes, only the steady state regime must simulated. In addition, the reactor is assumed to work near its set-point $\left(1600-1700^{\circ} \mathrm{C}\right)$. Therefore material properties dependency on the temperature was neglected. As a consequence, it will be shown that a weakly coupled multi-physics model is obtained.

All simulations are carried out in the 2D axisymmetric domain, which provided fairly good approximations when compared to 3D models, as demonstrated in [15].

Remark 1. Differential operators such as the gradient or the Laplacian to appear in this paper will refer to a cylindrical coordinate system, although no distinct notation will be used. Cylindrical coordinates are denoted by $(r, \theta, z)$ and their corresponding unit vectors as $\left(\mathbf{e}_{r}, \mathbf{e}_{\theta}, \mathbf{e}_{z}\right)$. The $2 D$ axisymmetric domain is therefore defined in the $(z, r)$ coordinates. 
In the following we synthetically review the equations governing the physics of the magnetic, thermal and flow fields.

\subsection{Magnetic Field Model}

The AC current flowing in the copper coil creates an alternating magnetic field which induces eddy currents in the electrically conductive parts of the reactor (graphite susceptor, mainly). The equation for the magnetic vector potential A is deduced from Maxwell equations for constant magnetic permeability, see [15] for details:

$$
\frac{1}{\mu_{0} \mu_{r}} \nabla^{2} \mathbf{A}=\mathrm{i} \omega \sigma \mathbf{A}-\mathbf{J}
$$

where $\mu_{0}$ is the magnetic permeability in vacuum, $\mu_{r}$ is the relative magnetic permeability of the material, $\omega=2 \pi f$ is the pulsation associated to the $\mathrm{AC}$ current frequency $f, \sigma$ is the electrical conductivity and $\mathbf{J}$ is the current density in the coil turns. The current density vector is oriented according to $\mathbf{e}_{\theta}$ direction: $\mathbf{J}=J_{\theta} \mathbf{e}_{\theta}$.

Remark 2. Since $J_{\theta}$ is the only non-zero component of the current density vector, Eq. (1) can be reduced to a scalar equation involving the $A_{\theta}$ component of the magnetic vector potential, $\mathbf{A}=A_{\theta} \mathbf{e}_{\theta}$, while $A_{r}$ and $A_{z}$ vanish.

Materials are assumed homogeneous and temperature-independent, so that the electrical resistivity of the coil and the electrical conductivity of the induced parts, $\varrho$ and $\sigma$ respectively, are constant and evaluated at the target temper-

ature. See Appendix in [15] for their respective constitutive relations. Once $\mathbf{A}$ is known, the induced heating source (i.e. the right-hand side of the heat equation) is computed from:

$$
Q=\frac{1}{2} \sigma \omega^{2}|\mathbf{A}|^{2}=\frac{1}{2} \sigma \omega^{2} \sqrt{\Re\left(A_{\theta}\right)^{2}+\Im\left(A_{\theta}\right)^{2}},
$$

where $\Re(\cdot)$ and $\Im(\cdot)$ denote the real and imaginary parts of a complex number, respectively.

\subsection{Flow Model}

The flow problem is modelled by incompressible steady state Navier-Stokes equations:

$$
\left\{\begin{array}{rl}
\rho_{g} \mathbf{u} \cdot \nabla \mathbf{u}-\eta \Delta \mathbf{u}+\nabla p & =\mathbf{0} \\
\nabla \cdot \mathbf{u} & =0
\end{array},\right.
$$

where $\mathbf{u}$ is the velocity field and $p$ is the pressure field. Both the gas density, $\rho_{g}$, and the gas dynamic viscosity, $\eta$, are assumed constant and independent of the temperature. 


\subsection{Heat Transfer Model}

Finally from the heat source $Q$ defined by Eq. (2) and the velocity $\mathbf{u}$ given by Eq. (3), the convection-diffusion heat equation can be written as follows:

$$
\rho C_{p} \mathbf{u} \cdot \nabla T-\lambda \nabla^{2} T=Q,
$$

being $\rho$ the density, $C_{p}$ the specific heat, $\lambda$ the thermal conductivity and $T$ the temperature field. These properties are considered homogeneous and independent of the temperature.

It is worth to remark that in general, the heat source $Q$ depends on the temperature field through the magnetic potential $\mathbf{A}$, as both the resistivity of the coil and the electrical conductivity of the induced parts are sensible to the temperature. Similarly, the velocity field $\mathbf{u}$ depends on the temperature through gas density and viscosity. Since all these material properties have been considered independent of the temperature, Eq. (1) and Eq. (3) are weakly coupled to Eq. (4), meaning that the temperature field depends on the velocity and the heat source, but these do not depend on temperature. This simplification is acceptable for preliminary design purposes. Further refinement of the model can be introduced if needed in a subsequent stage.

\section{Simulation of the parametrised multi-physics model}

As indicated in the previous section, a reduced order model of the reactor is built in this section on the basis of the multi-physics modelling introduced in section 2 .

We consider as design parameters the AC frequency (in fact, its associated the pulsation $\omega$ ), the electrical input power $P$ and the gas flow rate $q$. Therefore, the parametric domain is denoted by:

$$
\Xi:=\Omega_{\omega} \times \Omega_{P} \times \Omega_{q}
$$

with the following ranges of variation: $\omega \in \Omega_{\omega} \equiv 2 \pi[20,70] \mathrm{kHz}, P \in \Omega_{P} \equiv$ $[2.3,2.9] \mathrm{kW}$ and $q \in \Omega_{q} \equiv[0,28.27] \mathrm{slm}$ (equivalent to a Reynolds number in the range $[0,100]$, as explained in subsection 3.2 .

A priori reduced order modelling methods will be applied in next sections in order to solve a parametrised multi-physics problem defined in a higher dimensional domain, $\Omega \times \Xi \subset \mathbb{R}^{5}$, where $\Omega$ is the $2 \mathrm{D}$ axisymmetric computational domain to be defined in subsection 3.1. The proposed methods make use of separated representations in order to ensure low storage requirements of the multi-parametric solution and low computational complexity of the algorithms.

\subsection{Geometry and Computational Domain}

The physical domain is sketched in Fig. 2, as well as the different subdomains, subdomain interfaces and domain boundaries. Subdomains are denoted by $\Omega_{i}$, 
with $i=1, \ldots, 6$, while subdomain interfaces and domain boundaries are denoted by $\Gamma_{i}$, with $i=1, \ldots, 16$. The whole physical domain is denoted by:

$$
\Omega:=\bigcup_{i=1}^{6} \Omega_{i} .
$$

Subdomain $\Omega_{2}$ was created in order to impose a far field condition for the magnetic potential equation, as explained in subsection 3.3 . Fig. 2 also shows finite element mesh composed of 14,908 triangular elements, generated using GMSH [30. The same mesh was used for solving the magnetic potential, fluid flow and heat transfer problems.

\subsection{Solution of the Flow Field Using Hierarchical Basis Collocation}

Considering the computational domain defined in Fig. 2, the dimensionless flow problem is set up as follows:

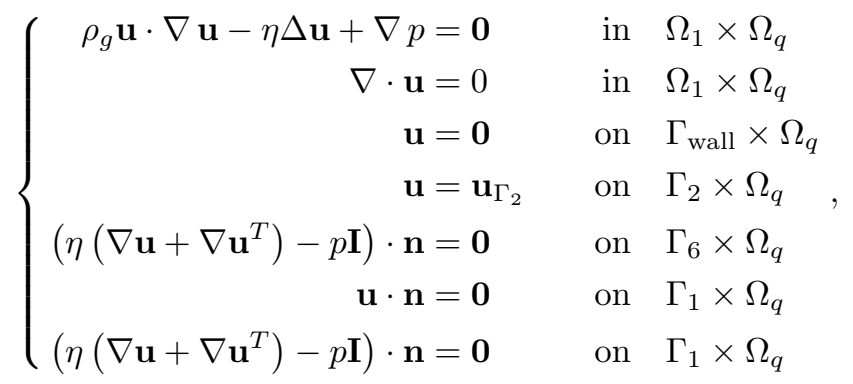

where $\Gamma_{\text {wall }}:=\bigcup_{i} \Gamma_{i}$ with $i=8,11, \ldots, 15$ and

$$
\mathbf{u}_{\Gamma_{2}}(r, q)=\frac{2\left(R_{\mathrm{in}}^{2}-r^{2}\right)}{\pi R_{\mathrm{in}}^{4}} q
$$

describes a fully developed Poiseuille profile, with $R_{\text {in }}$ the radius of the inlet section. A separated variables representation of the velocity field is sought:

$$
\mathbf{u}=\sum_{m=1}^{M_{u}} \alpha_{m} \boldsymbol{\psi}^{m} \quad \text { with } \quad \boldsymbol{\psi}^{m}(z, r, q):=\boldsymbol{\psi}_{s}^{m}(z, r) \psi_{q}^{m}(q),
$$

where $\boldsymbol{\psi}^{m}$ are the approximation basis functions, expressed as the product of vector space functions, $\boldsymbol{\psi}_{s}^{m}$, and scalar parameter functions, $\psi_{q}^{m}$, while $\alpha_{m}$ are the basis coefficients, which are uniquely determined for unit norm basis functions.

Space functions are approximated in $\Omega_{1}$ using Crouzeix-Raviart finite elements [31, which are second order accurate for the velocity field. The parametric space $\Omega_{q}$ is discretised using a hierarchical collocation approach. The collocation points are chosen as the Gauss-Chebyshev-Lobatto (GCL) abscissae. This set of points enjoys the nesting property and allows the definition of 


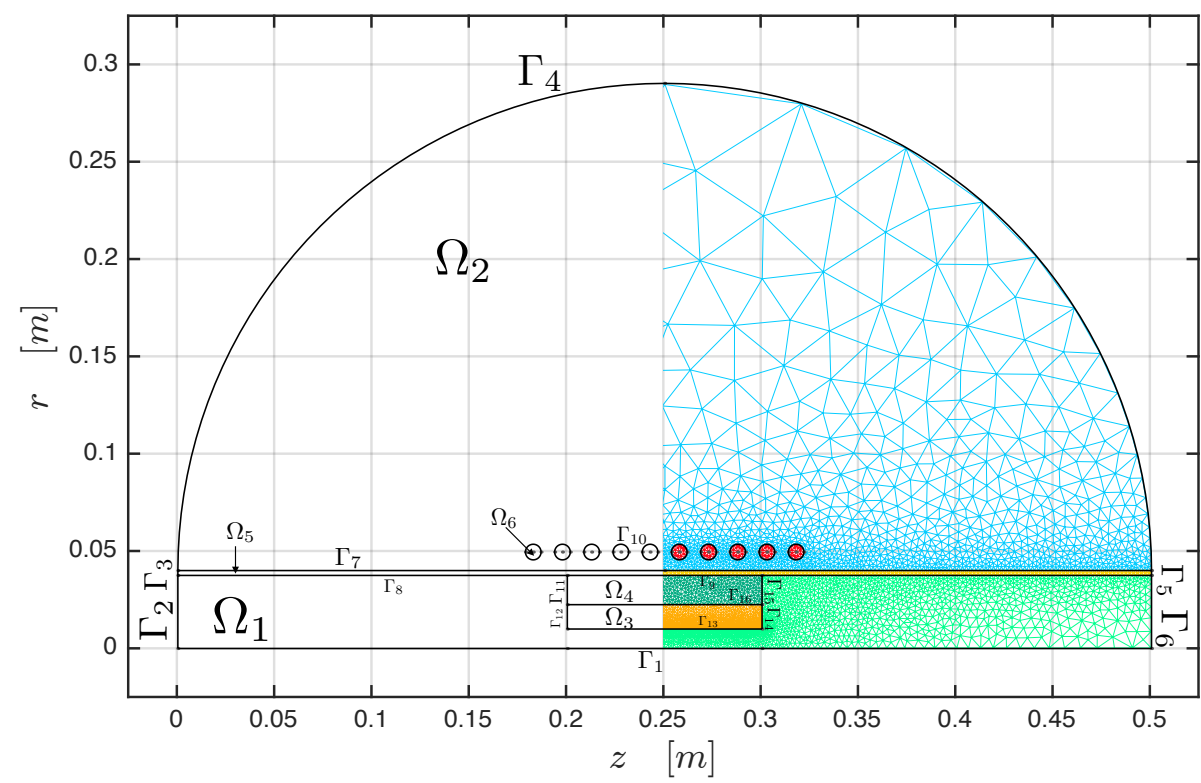

Figure 2: Two-dimensional axisymmetric computational domain of the CVD reactor: geometry (left half) and finite element mesh (right half). Subdomains definition: reactor chamber $\left(\Omega_{1}\right)$, void space around the quartz tube $\left(\Omega_{2}\right)$, graphite susceptor block $\left(\Omega_{3}\right)$, insulation coating block $\left(\Omega_{4}\right)$, quartz tube $\left(\Omega_{5}\right)$ and copper coil turns $\left(\Omega_{6}\right)$. Boundaries and interfaces between subdomains: axis of symmetry $\left(\Gamma_{1}\right)$, gas inlet/outlet $\left(\Gamma_{2} / \Gamma_{6}\right)$, quartz tube lateral boundaries $\left(\Gamma_{3}, \Gamma_{5}\right)$, quartz tube external wall $\left(\Gamma_{7}\right)$, quartz tube internal wall $\Gamma_{8}$, quartz-insulation interface $\left(\Gamma_{9}\right)$, coil turns boundary $\left(\Gamma_{10}\right)$, chamber-insulation interfaces $\left(\Gamma_{11}, \Gamma_{15}\right)$, chamber-graphite interfaces $\left(\Gamma_{12}, \Gamma_{13}, \Gamma_{14}\right)$ and insulationgraphite interface $\left(\Gamma_{16}\right)$. 


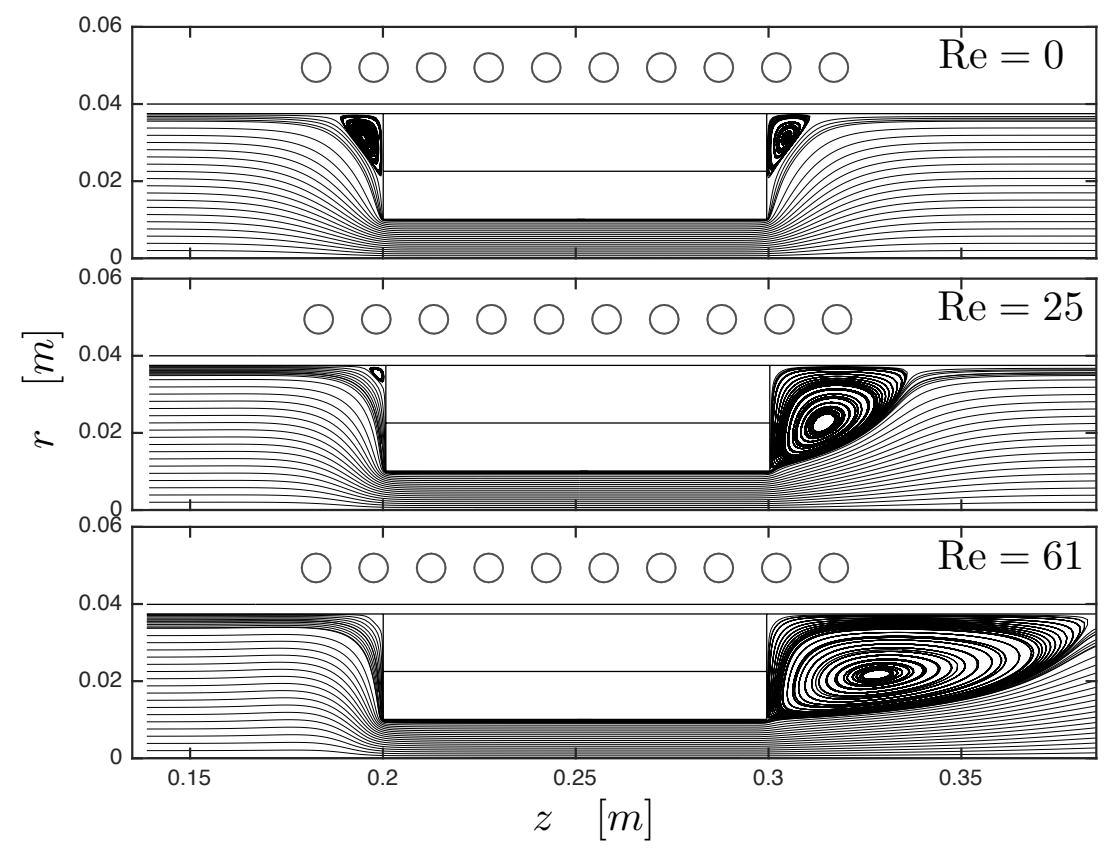

Figure 3: Streamlines of the flow field in the CVD reactor chamber for $R e=0$ (top), $R e=25$ (middle), $R e=61$ (bottom). 
a hierarchical basis with built-in error estimation capabilities. By consequence, the functions $\psi_{q}^{m}(q)$ are the hierarchical Lagrangian polynomials defined on the hierarchy of GCL points. The problem is solved using a greedy strategy consisting in recursively expanding the approximation space spanned by the polynomial functions by adding all the polynomials of a new hierarchy level and using collocation to determine the corresponding "surplus" functions $\boldsymbol{\psi}_{s}^{m}(z, r)$. One advantage of the collocation approach is that it uses a standard finite element solver to compute flow solutions corresponding to particular values of the parameter. Therefore, incompressibility can be straightforwardly enforced for parametric solutions. A more detailed illustration of the methodology is given in Appendix A, while a generalisation of the proposed strategy for the multiparametric case can be obtained using the Sparse Grid approach to circumvent the curse of dimensionality [32, 33, 34.

The adopted nonlinear solver for the Navier-Stokes equation is based on Picard iteration. Note that even though the solver outputs both velocity and pressure fields, only velocity is needed to couple the flow and heat transfer models. Convergence is reached when the solution residuals are less than $10^{-10}$, while the enrichment loop is terminated when the maximum norm of the hierarchical surpluses falls below $10^{-8}$. With this values the overall convergence is reached within 6 hierarchy levels, or equivalently, $M_{u}=33$ terms. Further compression of the solution is achieved by using the Singular Value Decomposition (SVD) over the set of hierarchical surpluses functions, reducing the number of terms to $M_{u}=25$. The scalar coefficients obtained from the projection of the hierarchical surpluses onto this new orthonormal basis are used to recombine the functions $\psi_{q}^{m}(q)$. Although inexpensive, this post-processing step is only relevant for the sake of compactness of the solution.

The dimensionless velocity field, defined as

$$
\mathbf{v}=\frac{\pi D^{2} \mathbf{u}}{4 q}
$$

where $D$ is the diameter of the smallest section of the reactor, is visualised in Fig. 3. The streamlines corresponding to different values of the Reynolds number, $\operatorname{Re}=\frac{4 \rho_{g} q}{\pi D \eta}$, are shown. It is worth to remark that these solutions are obtained by performing an inexpensive evaluation of the parametric solution, Eq. (6), since the velocity field is explicitly available as function of the gas flow rate.

\subsection{Solution of the Magnetic Field using PGD}

In this section, the heat source induced in the graphite susceptor is computed in terms of two parameters: the AC frequency and the electrical input power. Since the heat source depends linearly on the electrical input power, the magnetic potential only needs to be computed in terms of the AC frequency for a unit electrical input power. Therefore, Eq. (1) is extended to the parametric domain 
as follows:

$$
\left\{\begin{aligned}
\frac{1}{\mu_{0} \mu_{r}} \nabla^{2} A_{\theta} & =\mathrm{i} \omega \sigma A_{\theta}-J_{\theta} & & \text { in } \Omega \times \Omega_{\omega} \\
A_{\theta} & =0 & & \text { on } \bigcup_{i=2}^{6} \Gamma_{i} \times \Omega_{\omega} .
\end{aligned}\right.
$$

PGD seeks an approximation of the magnetic potential $A_{\theta}$ in a space-frequency separated representation:

$$
A_{\theta}=\sum_{m=1}^{M_{A}} \beta_{m} \phi^{m} \quad \text { with } \quad \phi^{m}(z, r, \omega):=\phi_{s}^{m}(z, r) \phi_{\omega}^{m}(\omega)
$$

where $\phi^{m}$ are the complex-valued basis functions, expressed as the product of space functions, $\phi_{s}^{m}$, and parameter functions, $\phi_{\omega}^{m}$. Both space and parameter functions are considered as complex-valued ${ }^{1}$. On the other hand, $\beta_{m}$ are realvalued basis coefficients, which are uniquely determined for unit norm basis functions. The PGD algorithm is based on a greedy enrichment algorithm which builds the separated representation Eq. (8) by adding one term at a time. Each new term is computed by solving a sequence of $2 \mathrm{D}$ and $1 \mathrm{D}$ problems to compute space and frequency functions, $\phi_{s}^{m}$ and $\phi_{\omega}^{m}$ respectively. Therefore, the method avoids the exponential complexity scaling characteristic of meshbased approaches to discretise the parametric domain. Details on the PGD formulation and implementation are given in Appendix B in order to keep the exposition of the results as clear as possible.

Space functions $\phi_{s}^{m}$ are approximated using $P 1$ triangular finite elements. Frequency-functions $\phi_{\omega}^{m}$ are approximated using linear 1D finite elements based on a discretisation of 101 equally spaced nodes in $\Omega_{\omega}$. The current density vector is also written as a space-frequency separated representation, consisting of a single functional pair:

$$
\begin{cases}J_{\theta}=J_{s}(z, r) J_{\omega}(\omega) & \text { in } \quad \Omega_{6} \times \Omega_{\omega}, \\ J_{\theta}=0 & \text { in } \quad\left(\Omega \backslash \Omega_{6}\right) \times \Omega_{\omega},\end{cases}
$$

with the following definition of the current density space-frequency functions:

$$
\begin{gathered}
J_{s}=\frac{1}{A_{\text {coil }}} \sqrt{P} \quad \text { and } \quad J_{\omega}=\frac{1}{\sqrt{R}}, \\
\text { with } \quad R=\frac{r_{\text {coil }} N}{D} \sqrt{2 \varrho \omega \mu_{0}},
\end{gathered}
$$

where $A_{\text {coil }}$ is the cross section area of the copper conductor, $P$ is the electrical input power and $R$ is the resistance which depends on the AC frequency due to the skin effect. Other magnitudes are: the coil radius, $r_{\text {coil }}$, the number of turns, $N$, and the copper electrical resistivity, $\varrho$.

\footnotetext{
${ }^{1}$ Notice that it is also possible to consider complex-valued space functions and real-valued parameter functions, and vice versa.
} 
The solution of the problem converged in $M_{A}=18$ terms, for a prescribed residual reduction of $10^{-8}$. The heat source could be then computed by performing a simple post-processing of the magnetic potential, as indicated in Eq. (2):

$$
Q=\sum_{m=1}^{M_{Q}} \gamma_{m} \zeta^{m} \quad \text { with } \quad \zeta^{m}(z, r, \omega):=\zeta_{s}^{m}(r, s) \zeta_{\omega}^{m}(\omega),
$$

where $\zeta^{m}$ are the basis functions expressed as the product of space functions, $\zeta_{s}^{m}$, and parameter functions, $\zeta_{\omega}^{m}$, while $\gamma_{m}$ are the basis coefficients, which are uniquely determined for unit norm basis functions. Eq. (9) was obtained with $M_{Q}=8$ basis functions, for a prescribed error of $10^{-8}$ using SVD. $\Omega_{P}$ is discretised using linear 1D finite elements with 101 equally spaced nodes. Since the heat source depends linearly on the electrical input power ${ }^{2}$, it is possible to include its dependency straightforwardly by simply redefining the heat source basis functions:

$$
\zeta^{m}(z, r, \omega, P):=\zeta_{s}^{m}(r, s) \zeta_{\omega}^{m}(\omega) \zeta_{P}^{m}(P) \quad \text { for } \quad 1 \leq m \leq M_{Q}
$$

since electrical input power functions are known beforehand: $\zeta_{P}^{m}(P) \equiv P$, for $1 \leq m \leq M_{Q}$.

Remark 3. Even if the heat source dependence on the electrical input power is trivial, the temperature field is not linear in this parameter because of the non homogeneous boundary conditions in the heat transfer problem, as defined in subsection 3.4 .

Fig. 4 shows the magnetic potential field lines in the central part of the reactor, for three different $\mathrm{AC}$ frequencies. The figure also shows the heat source induced in the graphite inductor. It can be observed that higher $\mathrm{AC}$ frequencies led to a less uniform heat source distribution inside the inductor, which tended to concentrate in the corners of the external boundary due to skin effect.

\subsection{Solution of Heat Transfer Problem using PGD}

This section describes the solution of the multi-parametric heat transfer model in terms of the AC frequency, the flow rate and the electrical input power. Eq. (4) is extended to the parametric domain:

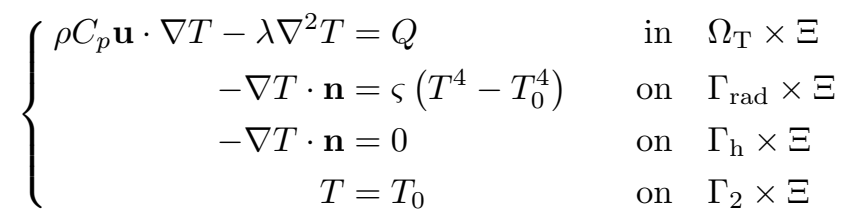

\footnotetext{
${ }^{2}$ The heat source is proportional to the square of the magnetic potential magnitude, which in turn is proportional to the square root of $P$ through the current density. Therefore, $Q$ is proportional to $P$.
} 


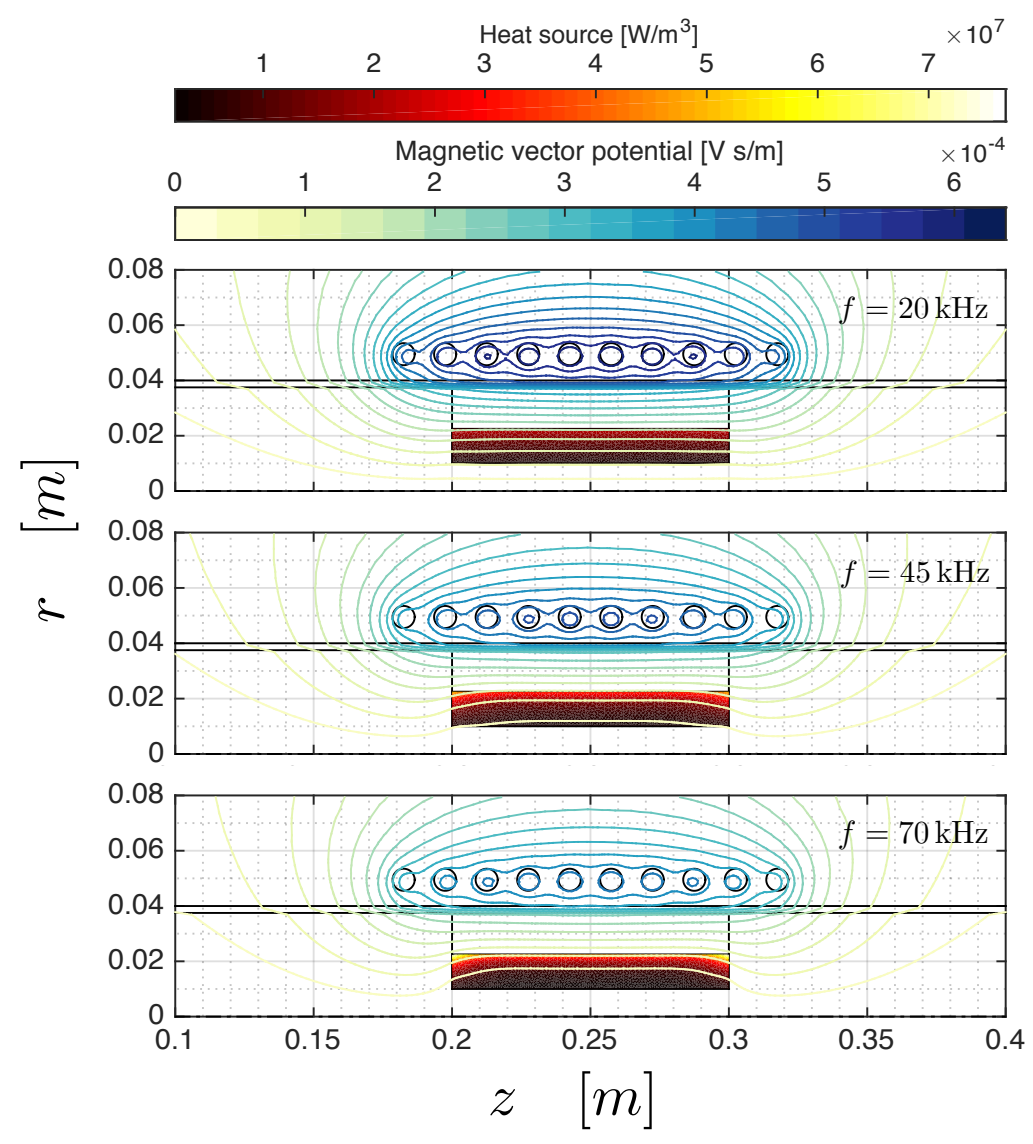

Figure 4: Contour plot of the magnetic potential field and induced heat source in the graphite susceptor for $f=\omega / 2 \pi=20 \mathrm{kHz}$ (top), $f=\omega / 2 \pi=45 \mathrm{kHz}$ (mid) and $f=\omega / 2 \pi=70 \mathrm{kHz}$ (bottom). 
where

$$
\Omega_{\mathrm{T}}=\bigcup_{i \in I_{\mathrm{T}}} \Omega_{i}, \quad \Gamma_{\mathrm{rad}}=\bigcup_{i \in I_{\mathrm{rad}}} \Gamma_{i} \quad \text { and } \quad \Gamma_{\mathrm{h}}=\bigcup_{i \in I_{\mathrm{h}}} \Gamma_{i}
$$

with $I_{\mathrm{T}}=(1,3,4,5), I_{\mathrm{rad}}=(7,11,12,13,14,15)$ and $I_{\mathrm{h}}=(1,3,5)$. The StefanBoltzmann constant is denoted by $\varsigma=5.67 \cdot 10^{-8} \mathrm{~W} / \mathrm{m}^{2} \mathrm{~K}^{4}$, while $T_{0}=298 \mathrm{~K}$ is the ambient temperature.

PGD is applied in order to compute a separated representation of the temperature field:

$$
\begin{aligned}
T & =\sum_{m=1}^{M_{T}} \kappa_{m} \varphi^{m}, \\
\text { with } \varphi^{m}(z, r, \omega, q, P): & =\varphi_{s}^{m}(z, r) \varphi_{\omega}^{m}(\omega) \varphi_{q}^{m}(q) \varphi_{P}^{m}(P),
\end{aligned}
$$

where $\varphi^{m}$ are basis functions expressed as the product of space functions, $\varphi_{s}^{m}$, frequency functions, $\varphi_{\omega}^{m}$, gas flow rate functions, $\varphi_{q}^{m}$, and electrical input power functions, $\varphi_{P}^{m}$. On the other hand, $\kappa_{m}$ are the basis coefficients, which are uniquely determined for unit norm basis functions. The procedure used to compute Eq. 12 is conceptually similar to the one adopted for the solution of the magnetic potential equation, discussed in Appendix B.

However, the nonlinearity introduced by the radiation term needs a particular treatment. Newton-Raphson (NR) algorithm is used to linearise the problem. At each nonlinear step, the associated Jacobian system is solved using PGD. Since the linearisation of the radiation term would involve the evaluation of $4 T^{3}$, assembling the corresponding Jacobian matrix would lead to a separated representation with up to $\left(M_{T}\right)^{3}$ operators. To overcome this difficulty, an approximated Jacobian using only $M_{T}^{\prime}<M_{T}$ terms is implemented. In practice, we consider $M_{T}^{\prime}=3$, which allows writing the Jacobian with 27 operators.

The resulting quasi-Newton algorithm converged in 8 iterations. The final solution consists of $M_{T}=75$ for a residual reduction of $10^{-5}$. The storage requirements of the separated variables representation are 8685 times lower than the ones corresponding to a full solution representation obtained by meshing the parametric space $\Xi$ with the equivalent resolution.

Fig. 5 shows the temperature field corresponding to specific points of the parametric space $\Xi$, evidencing the effect of the process parameters $\omega, q$ and $P$ on the temperature distribution inside the reactor.

\section{Process optimisation}

One of the notable advantages of explicitly computing parametric solutions of PDEs is that many applications such as control, inverse identification and optimisation can be performed very easily, because function evaluations are fairly inexpensive and can be obtained at the cost of a simple post-processing operations needed for the particularisation of the parametric solution. 


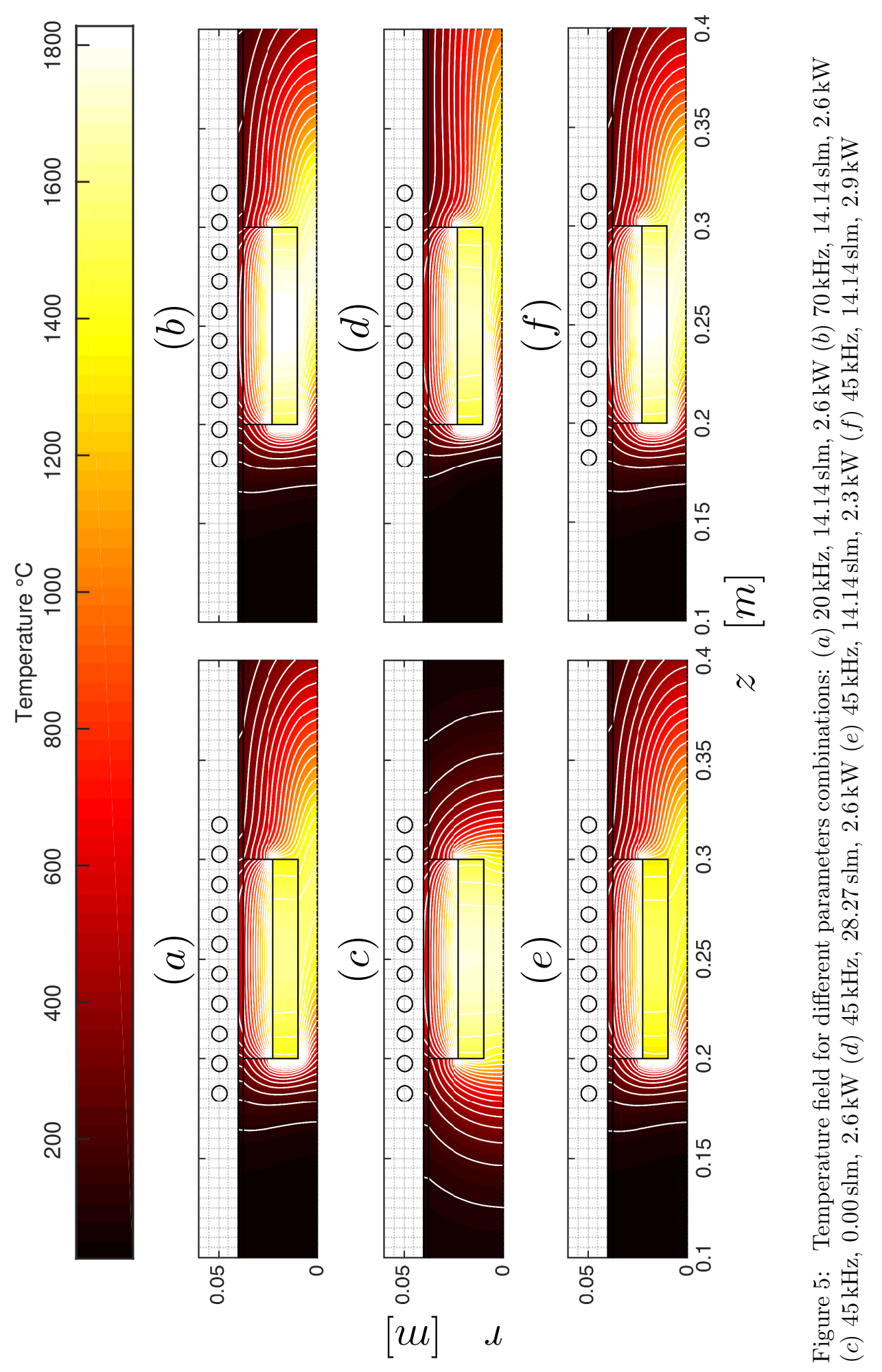


For this reason, ROM and in particular PGD have been widely used in the context of parameter optimisation in material processing techniques [35.

For CVD, optimisation often involves two main objectives. The first is the average temperature on the substrate where the film is grown, which should be as close as possible to the optimal chemical reaction temperature, $T_{t}$, while the other is the uniformity of the temperature distribution on the substrate, which directly affects the quality of the film. These two can be expressed through the following objective functions:

$$
f_{1}(\omega, q, P)=\left|1-\frac{\langle T\rangle}{T_{t}}\right| \quad \text { and } \quad f_{2}(\omega, q, P)=\frac{\sqrt{\left\langle(T-\langle T\rangle)^{2}\right\rangle}}{\langle T\rangle} .
$$

In Eq. $(13)$, the operator $\langle\cdot\rangle$ defines the average over a specified target surface of area $S_{t}$ :

$$
\langle T\rangle=\frac{1}{S_{t}} \int_{\Gamma_{13}} T(z, r, \omega, q, P) d z,
$$

where the target surface corresponds to the chamber-graphite horizontal wall. The Pareto front generated by these two objective functions is represented in Fig. (6). Each point in the grey shaded area is associated to a point in the parametric space $\Xi$ and therefore to a particular solution of the multi-physics problem. The Pareto front is simply generated by a cloud of one million points spanning the parametric space, since objective functions $f_{1}$ and $f_{2}$ are extremely cheap to evaluate once the parametrised solution is known. This construction, which may be very expensive when using standard simulation techniques, is carried out here in few seconds because any possible scenario has been computed offline.

Other objectives include for example the attainment of a specific flow rate $q_{t}$, which is dictated by the chemical reaction characteristic time, and the minimisation of the input power $P$, which obeys to an economical criterion. All these can be combined into a single multi-objective function:

$$
\begin{aligned}
C(\omega, q, P) & =c_{1}\left|1-\frac{\langle T\rangle}{T_{t}}\right|+c_{2} \frac{1}{\langle T\rangle} \sqrt{\left\langle(T-\langle T\rangle)^{2}\right\rangle}+ \\
& +c_{3} \frac{\left|q_{t}-q\right|}{q_{\max }-q_{t}}+c_{4} \frac{P-P_{\min }}{P_{\max }-P_{\min }} .
\end{aligned}
$$

The coefficients $c_{i}, i=1, \ldots, 4$ are the weights of the multi-objective optimisation.

We emphasise that the choice of the multi-objective function and its weights depend on the specific application and may vary from user to user and even evolve in time, whereas the parametric solution $T(z, r, \omega, q, P)$, describing the physical behaviour of the system, is invariant and may be reused if a new optimisation problem arises. Eq. (15) is a rather academic example that is only presented here to show the viability of the proposed approach, which essentially 


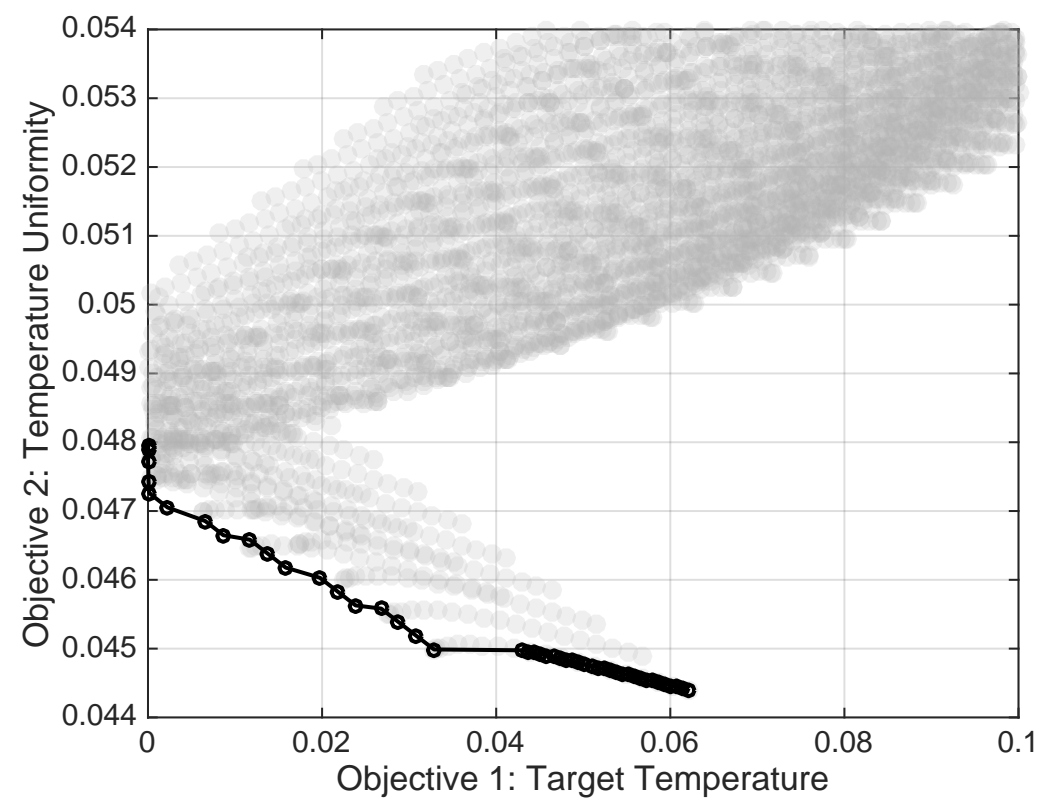

Figure 6: Pareto front. Objective 1 is related to the average temperature on the graphite susceptor with a target $T=1700^{\circ} \mathrm{C}$ and Objective 2 is related to the uniformity of the temperature distribution. 


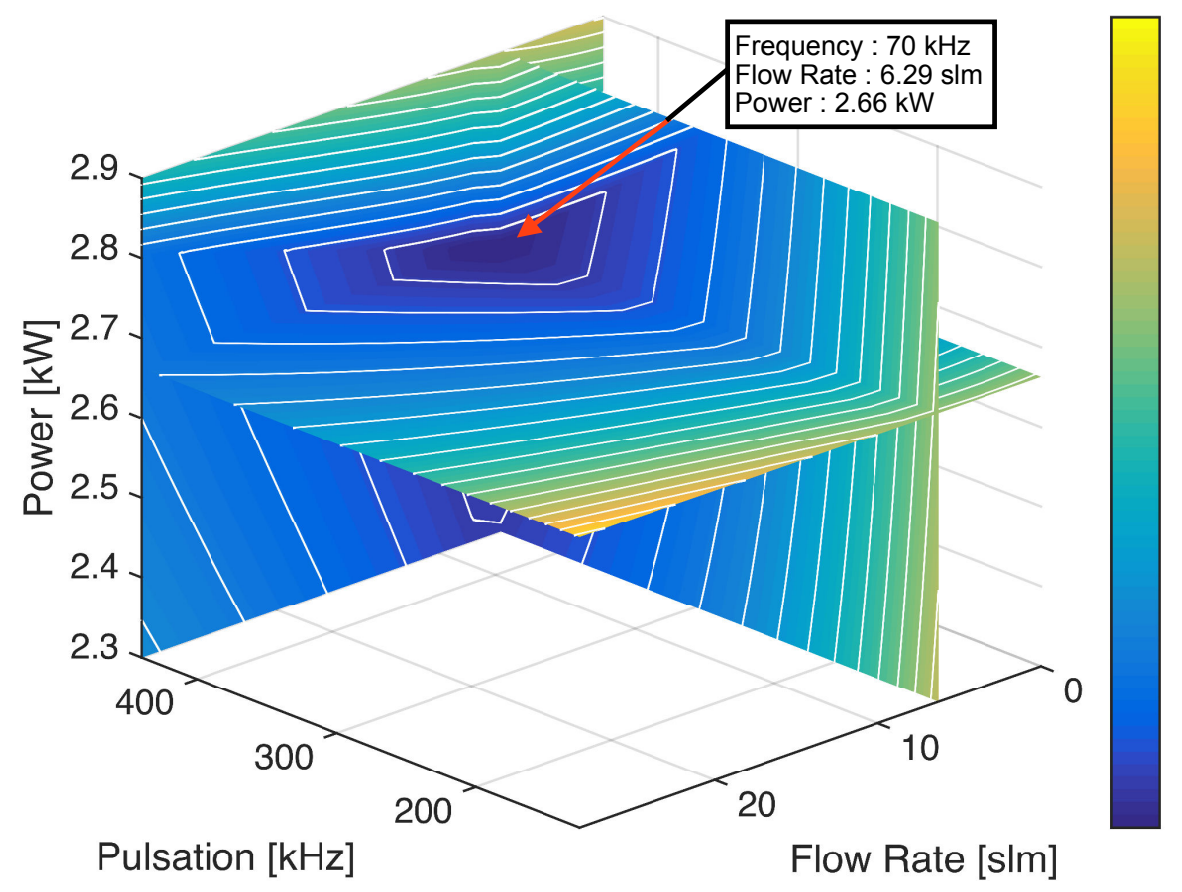

Figure 7: Plane slices visualisation of the objective function $C(\omega, q, P)$ corresponding to the coordinates of the optimal design point.

keeps the same complexity and structure even when more sophisticated and realistic objective functions are considered.

Given the weights $c_{1}=0.1089, c_{2}=0.8890, c_{3}=0.0012, c_{4}=8.12 \cdot 10^{-4}$, the target temperature $T_{t}=1700^{\circ} \mathrm{C}$ and the target flow rate of $q_{t}=8 \mathrm{slm}$, the function $C(\omega, q, P)$ is represented in figure 7. Two-dimensional slices corresponding to the planes $\omega / 2 \pi=70 \mathrm{kHz}, q=6.29 \mathrm{slm}$ and $P=2.66 \mathrm{~kW}$ are also shown. This coordinates correspond to the position of the optimal point. The minimisation of the multi-objective function $C(\omega, q, P)$ was performed through a simple gradient-based optimisation procedure. This can be performed practically in real time using MATLAB ${ }^{\circledR}$ routines. Note that there is no restriction in the choice of the optimisation algorithm that can be selected based on the particular application at hand.

Finally, the optimal solution is displayed in Fig. 8 showing the magnetic potential, flow and temperature fields.

\section{Conclusions}

In this paper we showed a computational strategy to produce reduced order solutions of the parametrised multi-physics equations governing the heat trans- 


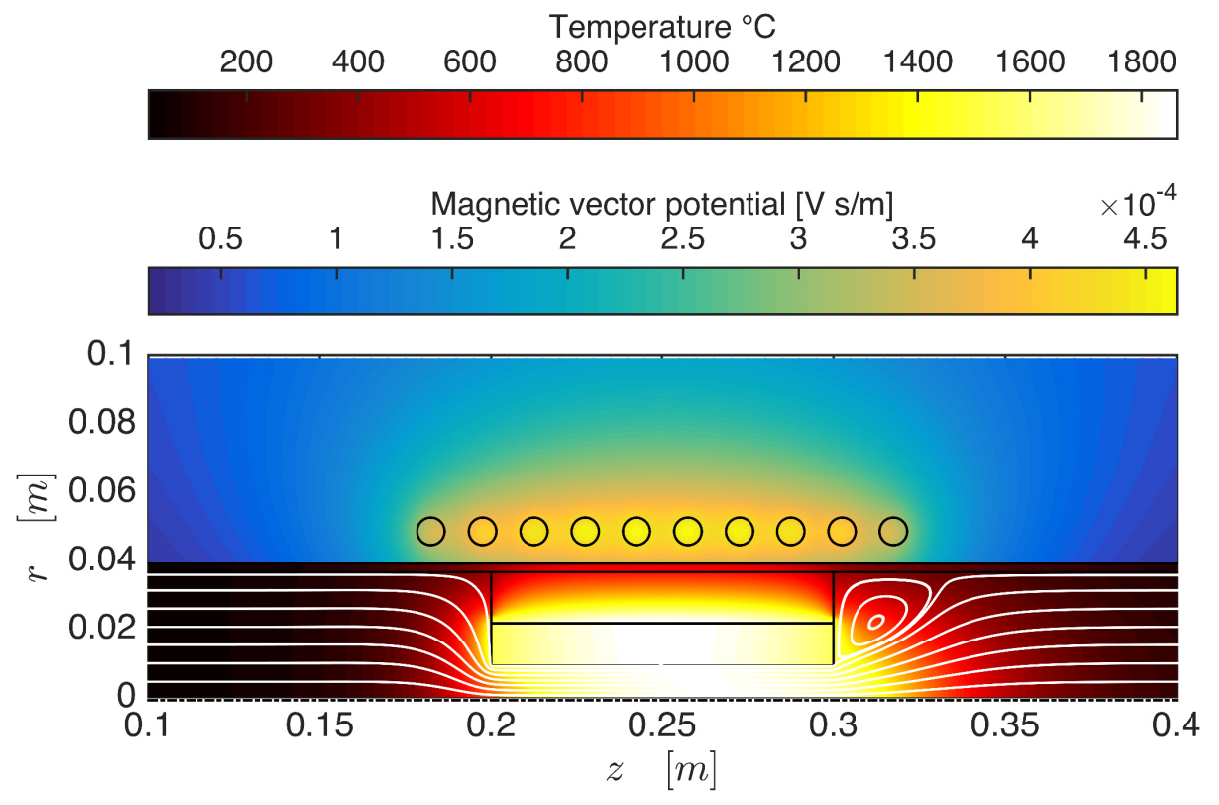

Figure 8: Magnetic potential field outside the reactor and flow and temperature fields inside the reactor, for the optimal parameter choice according to multiobjective optimisation function $C(\omega, q, P)$. 
fer in a hot-wall CVD reactor. Process parameters were optimised to obtain the most favourable process conditions. The approach that was followed involves two stages: the "off-line" computation of the parametric solution and the "on-line" optimisation. In the off-line stage the solution is computed using two different ROM techniques, PGD and hierarchical basis collocation, both providing a solution in a separated variables representation. This format provides advantages in terms of both storage requirements of the solution and easiness of manipulation. Indeed, once the solution is known, evaluating a quantity of interest and its gradient with respect to the parameters at any point of the parametric space becomes inexpensive and allows for fast optimisation. Further developments based on the strategy proposed in this paper include the possibility of performing sensitivity analysis and uncertainty quantification on the input parameters in order to assess the practical viability and the risks of the parameter combination that is find through optimisation. These two operations also benefit from the use of separated variables representations. The same formulation can include not only material and process parameters but also geometry and boundary conditions. These two ideas constitute part of the ongoing work and will be object of a future publication.

\section{A Separated representations based on Hierar- chical Basis Collocation}

A separated representation of the parametric velocity field, as in Eq. (6) can be obtained to an arbitrary accuracy and in a non-intrusive way, using the concept of hierarchical basis. According to the collocation approach the scalar parameter functions $\psi_{q}^{m}(q)$ are given a priori, while the vector space functions $\boldsymbol{\psi}_{s}^{m}(z, r)$ are to be determined by solving Eq. (5) in appropriate points of the parametric space named collocation points. The choice of using the same scalar parametric functions for both velocity components ensures that incompressibility is automatically enforced for any choice of the parameter, since vector space functions are divergence-free. The key of separated representation approximation based on collocation is the use of hierarchical basis.

There are many ways to define a hierarchical basis. In the following, polynomial interpolation is used. This guarantees high order accuracy provided that the function $\mathbf{u}(z, r, q)$ is sufficiently regular with the parameter $q$, which is the case for low Reynolds laminar flow. The collocation points are naturally chosen as the GCL points,

$$
\mathcal{P}_{n}=\left\{q_{0}, q_{1}, \ldots, q_{n}\right\},
$$

comprising the extrema of the $n$-th order Chebyshev polynomial of the first kind, $T_{n}(q)$, defined in $\Omega_{q} \equiv\left[q_{\min }, q_{\max }\right]$, plus the ending points: $q_{0}=q_{\min }$ and $q_{n}=q_{\max }$. GCL points have the important property of being "nested", in the sense that:

$$
\cdots \subset \mathcal{P}_{2(k-1)} \subset \mathcal{P}_{2 k} \subset \mathcal{P}_{2(k+1)} \subset \ldots \quad \text { for } \quad k \in \mathbb{N} .
$$


The 0-th level of the grid hierarchy only includes the ending points of the parametric space allowing for a linear approximation. Therefore:

$$
\begin{array}{rlrl}
\psi_{s}^{1}(z, r) & \equiv \mathbf{u}\left(z, r ; q_{\min }\right), & \psi_{q}^{1}(q)=\frac{q_{\min }-q}{q_{\min }-q_{\max }} \\
\psi_{s}^{2}(z, r) \equiv \mathbf{u}\left(z, r ; q_{\max }\right), & \psi_{q}^{2}(q)=\frac{q_{\max }-q}{q_{\max }-q_{\min }} .
\end{array}
$$

Each subsequent level $k$ of the hierarchy is constructed using the following procedure:

- The parametric functions $\psi_{q}^{m}(q)$ are identified with the Lagrangian polynomials:

$$
\mathscr{L}_{i}^{k}(q)=\frac{\prod_{j \neq i}\left(q-q_{j}\right)}{\prod_{j \neq i}\left(q_{i}-q_{j}\right)},
$$

with

$$
q_{i} \in \mathcal{P}_{2 k} \backslash \mathcal{P}_{2(k-1)} \quad \text { and } \quad q_{j} \in \mathcal{P}_{2 k} .
$$

- The corresponding surplus functions are determined as the difference between the solution computed for the new collocation points, $q_{i}$, and the approximation given by the interpolation from the previous hierarchical level, $\mathbf{u}^{k-1}$. Hence:

$$
\psi_{s}^{m}(z, r)=\mathbf{u}\left(z, r ; q_{i}\right)-\mathbf{u}^{k-1}\left(z, r, q_{i}\right) .
$$

This implies that the functions $\boldsymbol{\psi}_{s}^{m}(z, r)$ are not simply the solution of NavierStokes equations at the specified flow rate, $q_{i}$, but represent the difference between two consecutive hierarchical levels. These hierarchical surplus functions also offer a natural way to assess the convergence of the hierarchical enrichment procedure, which is stopped when the norm of all the newly added $\boldsymbol{\psi}_{s}^{m}(z, r)$ functions in a level is smaller than a desired tolerance. Theoretical error bounds can be found in 36 .

When the problem has more than one parameter, the same strategy can be paired with Smolyak's technique to generate Sparse Grids (SG) from the tensor product of one dimensional grids without incurring in the curse of dimensionality. This method retains the same convergence rate up to a logarithmic factor provided that the function has smooth high order mixed derivatives [37. More sophisticated variants include the possibility of dimensional adaptiveness [38] and local refinement [39] for a more efficient handling of high dimensional problems.

A concurrent but similar approach is represented by the Reduced Basis Method (RBM) 22, 40, 41. The substantial difference is that RBM is an "off-line" strategy that produces a reduced order model that can be efficiently 
solved "on-line" for a given choice of the model parameters, whereas the SG approaches produce a fully parametric solution that only needs to be particularised for the parameters of choice using interpolation. Another difference is the way these techniques handle nonlinearity which is completely straightforward in the case SG, whereas it needs a careful treatment for RBM by means, for instance, of the Empirical Interpolation Method [42. Thus, even if for a given order RBM produces more accurate solutions, SG has the advantage of its inherent offline/online nature. By considering a higher order approximation in the offline stage, the online evaluation of the solution could have the same accuracy that the one obtained by RBM based on lower order approximations.

\section{B PGD formulation of the magnetic potential problem}

Consider the weighted residual formulation of Eq. (7):

$$
a\left(A_{\theta}, A_{\theta}^{*}\right)+b\left(A_{\theta}, A_{\theta}^{*}\right)=\ell\left(A_{\theta}^{*}\right),
$$

where $A_{\theta}^{*}$ is a test function chosen in the appropriate functional space. Bilinear and linear forms are defined as follows:

$$
\begin{aligned}
a\left(A_{\theta}, A_{\theta}^{*}\right) & :=\int_{\Omega \times \Omega_{\omega}} i \omega \sigma \bar{A}_{\theta}^{*} A_{\theta} r d z d r d \omega, \\
b\left(A_{\theta}, A_{\theta}^{*}\right) & :=\int_{\Omega \times \Omega_{\omega}} \frac{1}{\mu_{0} \mu_{r}} \nabla \bar{A}_{\theta}^{*} \cdot \nabla A_{\theta} r d z d r d \omega \text { and } \\
\ell\left(A_{\theta}^{*}\right) & :=\int_{\Omega \times \Omega_{\omega}} \bar{A}_{\theta}^{*} J_{\theta} r d z d r d \omega,
\end{aligned}
$$

where we denote the complex conjugate of $v \in \mathbb{C}$ by $\bar{v}$ in order to define an appropriate complex inner product. PGD builds the space-frequency separated representation of the magnetic potential field, Eq. (8), by adding one basis function at a time. Suppose that $k-1<M_{A}$ terms are already known and the $k$-th wants to be computed:

$$
A_{\theta}=\sum_{m=1}^{k-1} \beta_{m} \phi^{m}+\phi .
$$

When the computation of $\phi$ converges (it involves a nonlinear problem as explained below), we set $\beta_{k}=\|\phi\|$ and $\phi^{k}=\phi / \beta_{k}$. Introducing Eq. (22) into Eq. (21):

$$
a\left(\phi, \phi^{*}\right)+b\left(\phi, \phi^{*}\right)=r^{k-1}\left(\phi^{*}\right),
$$

where $r^{k-1}$ denotes the residual corresponding to the magnetic potential approximation with $k-1$ terms. Hence:

$$
r^{k-1}\left(\phi^{*}\right):=\ell\left(\phi^{*}\right)-\sum_{m=1}^{k-1} \beta_{m}\left[a\left(\phi^{m}, \phi^{*}\right)+b\left(\phi^{m}, \phi^{*}\right)\right] .
$$


PGD seeks a separated variables representation of $\phi$, meaning that:

$$
\phi(z, r, \omega):=\phi_{s}(z, r) \phi_{\omega}(\omega) .
$$

Observe that the computation of both $\phi_{s}$ and $\phi_{\omega}$ involves solving a nonlinear problem, as both unknowns multiply each other. This is classically solved by using an alternating directions strategy, meaning that both functions are sequentially updated until convergence.

1. Suppose that $\phi_{\omega}$ is known (in practice, it is initialised randomly). As a consequence, the only possible variation is in the space function, which allows writing the test function as follows: $\phi^{*}=\phi_{s}^{*} \phi_{\omega}$. Substituting in Eq. 23):

$$
a\left(\phi_{s} \phi_{\omega}, \phi_{s}^{*} \phi_{\omega}\right)+b\left(\phi_{s} \phi_{\omega}, \phi_{s}^{*} \phi_{\omega}\right)=r^{k-1}\left(\phi_{s}^{*} \phi_{\omega}\right)
$$

Then, it is possible to update $\phi_{s}$ by solving a space problem defined in the $2 \mathrm{D}$ axisymmetric domain:

$$
v_{\omega} a_{s}\left(\phi_{s}, \phi_{s}^{*}\right)+\tau_{\omega} b_{s}\left(\phi_{s}, \phi_{s}^{*}\right)=r_{s}^{k-1}\left(\phi_{s}^{*}\right)
$$

with the following definition of the right-hand side:

$$
\begin{aligned}
r_{s}^{k-1}\left(\phi_{s}^{*}\right) & :=\vartheta_{\omega} \ell_{s}\left(\phi_{s}^{*}\right)- \\
& -\sum_{m=1}^{k-1} \beta_{m}\left[v_{\omega}^{m} a_{s}\left(\phi_{s}^{m}, \phi_{s}^{*}\right)+\tau_{\omega}^{m} b_{s}\left(\phi_{s}^{m}, \phi_{s}^{*}\right)\right]
\end{aligned}
$$

Linear and bilinear forms have been split into their space and frequency parts according to the following definitions:

$$
\begin{aligned}
a_{s}\left(\phi_{s}, \phi_{s}^{*}\right) & :=\int_{\Omega} i \sigma \bar{\phi}_{s}^{*} \phi_{s} r d z d r, & a_{\omega}\left(\phi_{\omega}, \phi_{\omega}\right) & :=\int_{\Omega_{\omega}} \omega \overline{\phi_{\omega}} \phi_{\omega} d \omega, \\
b_{s}\left(\phi_{s}, \phi_{s}^{*}\right) & :=\int_{\Omega} \frac{1}{\mu_{0} \mu_{r}} \nabla \overline{\phi_{s}^{*}} \cdot \nabla \phi_{s} r d z d r, & b_{\omega}\left(\phi_{\omega}, \phi_{\omega}\right) & :=\int_{\Omega_{\omega}} \bar{\phi}_{\omega} \phi_{\omega} d \omega, \\
\ell_{s}\left(\phi_{s}^{*}\right) & :=\int_{\Omega} \bar{\phi}_{s}^{*} J_{s} r d z d r, & \ell_{\omega}\left(\phi_{\omega}\right) & :=\int_{\Omega_{\omega}} \bar{\phi}_{\omega} J_{\omega} d \omega .
\end{aligned}
$$

Using the previous definitions, scalar coefficients introduced in Eq. 26 and Eq. 27) are computed as follows:

$$
\begin{aligned}
& v_{\omega}=a_{\omega}\left(\phi_{\omega}, \phi_{\omega}\right), \quad \tau_{\omega}=b_{\omega}\left(\phi_{\omega}, \phi_{\omega}\right), \quad \vartheta_{\omega}=\ell_{\omega}\left(\phi_{\omega}\right), \\
& v_{\omega}^{m}=a_{\omega}\left(\phi_{\omega}^{m}, \phi_{\omega}\right), \quad \tau_{\omega}^{m}=b_{\omega}\left(\phi_{\omega}^{m}, \phi_{\omega}\right), \quad 1 \leq m \leq k-1 \text {. }
\end{aligned}
$$

2. From $\phi_{s}$ just computed using Eq. 26, the frequency function $\phi_{\omega}$ can be updated. The test function writes in this case as follows: $\phi^{*}=\phi_{s} \phi_{\omega}^{*}$. 
Substituting and operating, it is possible to update $\phi_{\omega}$ by solving a 1D problem defined in the frequency domain:

$$
v_{s} a_{\omega}\left(\phi_{\omega}, \phi_{\omega}^{*}\right)+\tau_{s} b_{\omega}\left(\phi_{\omega}, \phi_{\omega}^{*}\right)=r_{\omega}^{k-1}\left(\phi_{\omega}^{*}\right),
$$

with the following definition of the right-hand side:

$$
\begin{aligned}
r_{\omega}^{k-1}\left(\phi_{\omega}^{*}\right) & :=\vartheta_{s} \ell_{\omega}\left(\phi_{\omega}^{*}\right)- \\
& -\sum_{m=1}^{k-1} \beta_{m}\left[v_{s}^{m} a_{\omega}\left(\phi_{\omega}^{m}, \phi_{\omega}^{*}\right)+\tau_{s}^{m} b_{\omega}\left(\phi_{\omega}^{m}, \phi_{\omega}^{*}\right)\right],
\end{aligned}
$$

where linear and bilinear forms already defined in Eq. (28) have been used. Scalar coefficients introduced in Eq. (29) and Eq. (30) are computed as follows:

$$
\begin{array}{rrrr}
v_{s}=a_{s}\left(\phi_{s}, \phi_{s}\right), & \tau_{s}=b_{s}\left(\phi_{s}, \phi_{s}\right), & \vartheta_{s}=\ell_{s}\left(\phi_{s}\right), \\
v_{s}^{m}=a_{s}\left(\phi_{s}^{m}, \phi_{s}\right), & \tau_{s}^{m}=b_{s}\left(\phi_{s}^{m}, \phi_{s}\right), & 1 \leq m \leq k-1 .
\end{array}
$$

A stagnation criterion is implemented in order to evaluate the convergence of the pair $\phi_{s} \phi_{\omega}$. The number of basis functions to be added to the approximation defined by Eq. 222 is determined using a measure of the residual reduction or an appropriate error estimator for separated representations [43].

\section{References}

[1] F. Zorriassatine, C. Wykes, R. Parkin, N. Gindy, A survey of virtual prototyping techniques for mechanical product development, Proc. Inst. Mech. Eng. B J. Eng. Manuf. 217(4) (2003) 513-530.

[2] K.L. Choy, Chemical vapour deposition of coatings, Prog. Mater. Sci. 48(2) (2003) 57-170.

[3] A.C. Jones, M.L. Hitchman, Chemical vapour deposition: precursors, processes and applications, The Royal Society of Chemistry, 2009.

[4] P.W. May, N.L. Allan, M.N. Ashfold, J.C. Richley, Y.A. Mankelevich, Simplified Monte Carlo simulations of chemical vapour deposition diamond growth, J. Phys. Condens. Mat. 21(36) (2009) 364203-364222.

[5] A.M. Ito, A. Takayama, S. Saito, N. Ohno, S. Kajita, H. Nakamura, Molecular dynamics simulation of chemical vapor deposition of amorphous carbon: dependence on H/C ratio of source gas, Jpn. J. Appl. Phys. 50(1S1) (2011) 01AB01.

[6] M. Grujicic, S.G. Lai, Atomistic simulation of chemical vapor deposition of (111)-oriented diamond film using a kinetic Monte Carlo method, J. Mater. Sci. 34 (1999) 7-20. 
[7] P.W. May, J.N. Harvey, N.L. Allan, J.C. Richley, Y.A. Mankelevich, Simulations of chemical vapor deposition diamond film growth using a kinetic Monte Carlo model, J. Appl. Phys. 108(11) (2010) 114909.

[8] C.C. Battaile, D.J. Srolovitza, J.E. Butler, A kinetic Monte Carlo method for the atomic-scale simulation of chemical vapor deposition: application to diamond, J. Appl. Phys. 82(12) (1997) 6293-6300.

[9] J. Houska, J.E. Klemberg-Sapieha, L. Martinu, Atom-by-atom simulations of chemical vapor deposition of nanoporous hydrogenated silicon nitride, J. Appl. Phys. 107(8) (2010) 083501.

[10] L. Meng, Q. Sun, J. Wang, F. Ding, Molecular dynamics simulation of chemical vapor deposition graphene growth on Ni (111) surface, J. Phys. Chem. C 116(10) (2012) 6097-6102.

[11] O.K.M. Hafiz, A. Singh, CFD simulation of laser enhanced modified chemical vapor deposition process, Chem. Eng. Res. Des. 89 (2011) 593-602.

[12] M.K. Gobbert, T.P. Merchant, L.J. Borucki, T.S. Cale, A multiscale simulator for low pressure chemical vapor deposition, J. Electrochem. Soc. 144(11) (1997) 3945-3951.

[13] D. Endres, S. Mazumder, Numerical investigation of pulsed chemical vapor deposition of aluminum nitride to reduce particle formation, J. Cryst. Growth, 335(1) (2011) 42-50.

[14] S.A. Konakov, V.V. Krzhizhanovskaya, A mathematical model and simulation results of plasma enhanced chemical vapor deposition of silicon nitride films, JPCS 574 (2015) 012144.

[15] O. Danielsson, U. Forsberg, A. Henry, E. Janzen, Investigation of the temperature profile in a hot-wall $\mathrm{SiC}$ chemical vapor deposition reactor, J. Cryst. Growth 235 (2002) 352-364.

[16] K. Choi J. Kim, CFD simulation of chemical vapor deposition of silicon carbide in $\mathrm{CH}_{3} \mathrm{SiCl}_{3}-\mathrm{H}_{2}$ system, Curr. Nanosci. 10 (2014) 135-137.

[17] J. Choo, R.A. Adomaitis, G.W. Rubloff, L. Henn-Lecordier, Y. Liu, Simulation-based design and experimental evaluation of a spatially controllable CVD reactor, AIChE J. 51(2) (2005) 572-584.

[18] M. Masi, C. Cavallotti, D. Boccalari, F. Castellana, Preliminary design of a novel high throughput CVD reactor for photovoltaic applications, Cryst. Res. Technol. 49(8) (2014) 614-619.

[19] A. Antoulas, D. Sorensen, S. Gugercin, A survey of model reduction methods for large-scale systems, Contemp. Math. 280 (2001) 193-220. 
[20] R.A. Bialecki, A.J. Kassab, A. Fic, Proper Orthogonal Decomposition and modal analysis for acceleration of transient FEM thermal analysis, Int. J. Numer. Meth. Engrg. 62(6) (2005) 774-797.

[21] C. Prud'homme, D. Rovas, K. Veroy, L. Machiels, Y. Maday, A.T. Patera, G. Turinici, Reliable real-time solution of parametrized partial differential equations: Reduced-Basis Output Bound Methods, J. Fluids Eng. 124(1) (2001) 70-80.

[22] Y. Maday, E.M. Ronquist, A reduced-basis element method, C. R. Acad. Sci. Paris Ser. I, 335 (2002) 195-200.

[23] F. Chinesta, A. Ammar, E. Cueto, Recent advances and new challenges in the use of the Proper Generalized Decomposition for solving multidimensional models, Arch. Comput. Methods Eng. 17(4) (2010) 327-350.

[24] F. Chinesta, R. Keunings, A. Leygue, The Proper Generalized Decomposition for advanced numerical simulations: a primer, Springer, 2014.

[25] F. Chinesta, P. Ladevèze, E. Cueto, A short review in model order reduction based on Proper Generalized Decomposition, Arch. Comput. Methods Eng. 18 (2011) 395-404.

[26] F. Chinesta, A. Leygue, F. Bordeu, J.V. Aguado, E. Cueto, D. Gonzalez, I. Alfaro, A. Ammar, A. Huerta, PGD-based Computational Vademecum for efficient design, optimization and control, Arch. Comput. Methods Eng. 20(1) (2013) 31-59.

[27] A. Theodoropoulou, R.A. Adomaitis, E. Zafiriou, Model reduction for optimization of rapid thermal chemical vapor deposition systems, IEEE T. Semiconduct. M. 11(1) (1998) 85-98.

[28] G.M. Kepler, H.T. Tran, H.T. Banks, Reduced order model compensator control of species transport in a CVD reactor, Optim. Contr. Appl. Meth. 21(4) (2000) 143-160.

[29] H.T. Banks, H.T. Tran, Reduced order based compensator control of thin film growth in a CVD reactor, In: Optimal Control of Complex Structures, Birkhäuser, 2001.

[30] C. Geuzaine, J.-F. Remacle, Gmsh: a three-dimensional finite element mesh generator with built-in pre- and post-processing facilities, Int. J. Numer. Meth. Engrg. 79(11) (2009) 1309-1331.

[31] J. Donea, A. Huerta, Finite element methods for flow problems, John Wiley \& Sons, 2003.

[32] H.J. Bungartz, M. Griebel, Sparse Grids, Acta Numer. 13 (2004) 147-269. 
[33] B. Peherstorfer, S. Zimmer, H.J. Bungartz, Model reduction with the Reduced Basis Method and Sparse Grids, In: Sparse Grids and Applications, Springer, 2012.

[34] A. Cohen, R. DeVore, Approximation of high-dimensional parametric PDEs, Acta Numer. 24 (2015) 1-159.

[35] F. Chinesta, E. Cueto, PGD-based modeling of materials, structures and processes, Springer, 2014.

[36] V. Barthelmann, E. Novak, K. Ritter, High dimensional polynomial interpolation on sparse grids, Adv. Comput. Math. 12(4) (2000) 273-288.

[37] S.A. Smolyak, Quadrature and interpolation formulas for tensor products of certain classes of functions, Dokl. Akad. Nauk SSSR 4 (1963) 240-243.

[38] T. Gerstner, M. Griebel, Dimension-adaptive tensor-product quadrature, Computing 71(1) (2003) 65-87.

[39] J.D. Jakeman, S.G. Roberts, Local and dimension adaptive stochastic collocation for uncertainty quantification, In: Sparse Grids and Applications, Springer, 2012.

[40] Y. Maday, A.T. Patera, G. Turinici, A priori convergence theory for reduced-basis approximations of single-parametric elliptic partial differential equations, J. Sci. Comput. 17(1-4) (2002) 437-446.

[41] G. Rozza, D.B.P. Huynh, A.T. Patera, Reduced basis approximation and a posteriori error estimation for affinely parametrized elliptic coercive partial differential equations: application to transport and continuum mechanics, Arch. Comput. Methods Eng. 15(3) (2008) 229-275.

[42] Y. Maday, N.C. Nguyen, A.T. Patera, S.H. Pau, A general multipurpose interpolation procedure: the magic points, CPAA 8(1) (2009) 383-404.

[43] A. Ammar, F. Chinesta, P. Díez, A. Huerta, An error estimator for separated representations of highly multidimensional models, Comput. Meth. Appl. Mech. Engrg. 199(25-28) (2010) 1872-1880. 


\section{About the authors \\ Domenico Borzacchiello, Jose V. Aguado and Francisco Chinesta}

Institut de Calcul Intensif (ICI) at Ecole Centrale de Nantes

1 rue de la Noë, BP 92101, 44321 Nantes cedex 3, France

e-mail: \{domenico.borzacchiello, jose.aguado-lopez, francisco.chinesta\}@ec-nantes.fr web: http://ici.ec-nantes.fr 